\title{
Synthesis of Deprenyl-like nitroxide free radicals and their diamagnetic derivatives
}

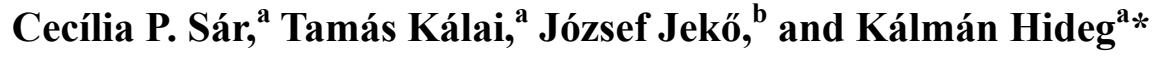 \\ ${ }^{a}$ Department of Organic and Medicinal Chemistry, University of Pécs, 7602 Pécs, P. O. Box 99, \\ Hungary \\ ${ }^{b}$ Department of Chemistry, College of Nyíregyháza, Sóstói st 31/B, H-4440, Nyíregyháza, \\ Hungary \\ E-mail: kalman.hideg@aok.pte.hu
}

This article is dedicated to Professor Ferenc Fülöp on occasion of his 60th birthday

\begin{abstract}
Synthesis of paramagnetically modified deprenyl and oxotremorine is reported. Starting from 5and 6-membered 2,5-disubstituted nitrones 1, 6 or 4-phenyl-2,5,5-trimethyl-1 $H$-pyrroline 1-oxide 11 deprenyl or oxotremorine like nitroxides were synthesized via Grignard reactions. The corresponding pre-nitroxides with propargylamine structure were achieved by reduction of nitroxides followed by methylation.
\end{abstract}

Keywords: Amines, alkylations, L-deprenyl, free radicals, Grignard reaction

\section{Introduction}

Parkinson's disease is an age-related disorder that afflicts as many as $2 \%$ of all individuals. ${ }^{1}$ The biochemical basis for the motor symptoms of Parkinson's disease is a loss of dopamine., Therefore Parkinson's disease can be relieved by treating patients with L-3,4dihydroxyphenylalanine (L-DOPA) and an inhibitor of peripheral L-DOPA decarboxylase. In order to preserve brain dopamine, it is also common to treat patients with a monoamine oxidase B (MAO-B) inhibitor. ${ }^{4}$ Selegiline (L-Deprenyl) and Rasagiline (Figure 1) 5-10 $^{5}$ are selective inhibitors of MAO-B and Selegiline is currently used for the treatment of Parkinson's and Alzheimer's diseases. This compound was reported to have a neuroprotective activity due to the prevention of apoptosis. ${ }^{11}$ The propargylamine pharmacophore of Selegiline and Rasagiline appears to be responsible for neuroprotective activity. Crystallographic analysis revealed that rasagiline covalently binds with its propargyl group to flavine enzyme to form an iminopropene chain. $^{12}$ 

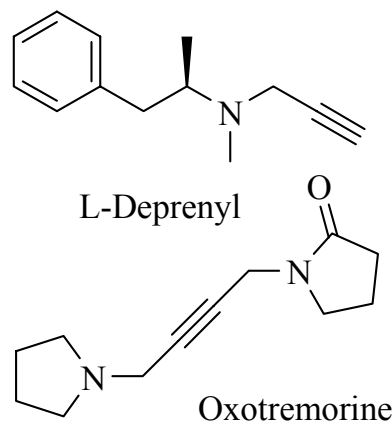

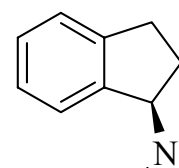

Rasagiline

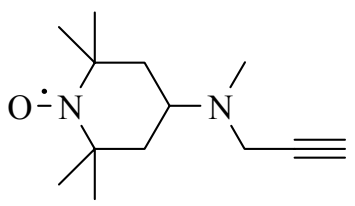

JSAK-648

\section{Figure 1}

Namiecinski et al. found that nitroxides with propargylamine chain such as JSAK-648 (Figure 1) can cross the blood-brain barrier and have been shown to have antioxidant properties, cell protection against oxidative stress and Reactive Oxygen Species (ROS) cytotoxicity. ${ }^{13,14}$ It is important to have a radical scavenger in statu nascendi to prevent damages caused by ROS, because the MAO-B mediated metabolism of dopamine and its autooxidation generate $\mathrm{O}_{2}{ }^{-}, \mathrm{H}_{2} \mathrm{O}_{2}$ and the highly toxic $\mathrm{OH}$ in the presence of trace levels of free iron ions. Continuing our research in the synthesis of experimental drugs with dual activity containing nitroxide or its precursor, ${ }^{15-19}$ we wish to extend this idea for neuroprotective drugs such as Deprenyl and Oxotremorine. Oxotremorine (Figure 1) is known as a muscarinic agonist and is also used in the Alzheimer's therapy. Therefore a large number of Oxotremorine derivatives were reported and investigated. $^{20-22}$

The metabolic oxidation of 1-methy-4-phenyl-1,2,3,6-tetrahydropyridine (MPTP) to the oxidation product $\mathrm{MPDP}^{+}$is also catalyzed by MAO-B. Following a second two-electron oxidation, the ultimate neurotoxic metabolite, 1-methyl-4-phenylpyridinium $\left(\mathrm{MPP}^{+}\right)$is generated. ${ }^{23,24} \mathrm{MPP}^{+}$is a mitochondrial toxin, which selectively damages nigrostriatal neurons and induces Parkinsonian syndrome in humans. ${ }^{25}$ Several other allylamines act as good MAO-B substrates, such as the 5-membered ring analogue of MPTP. ${ }^{23}$ (Figure 2) 

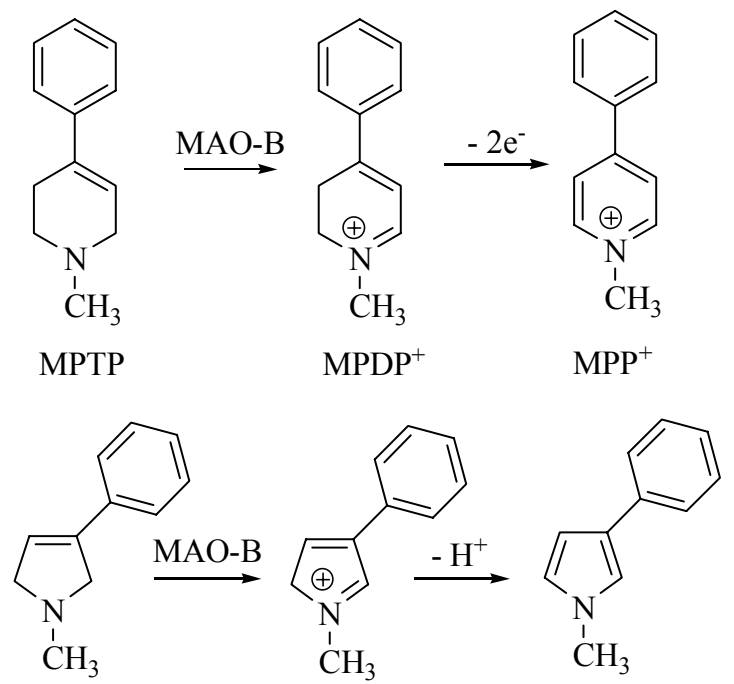

\section{Figure 2}

Motivated by these findings the synthesis of Deprenyl and Oxotremorine derivatives with 5- or 6-membered nitroxide rings was planned. We desired to investigate the oxidation of their sterically hindered secondary or tertiary amine precursors and a six membered model compound. We hope that these compounds will act as MAO-B antagonists or muscarinic agonists and as antioxidant compounds in the central nervous system (CNS).

\section{Results and Discussion}

An evident approach for the combination of Deprenyl and Oxotremorine structure with nitroxides was the reaction of five- or six-memberd nitrones and properly chosen Grignardreagents, supporting the introduction of the required aryl or alkynyl group easily (Figure 3).

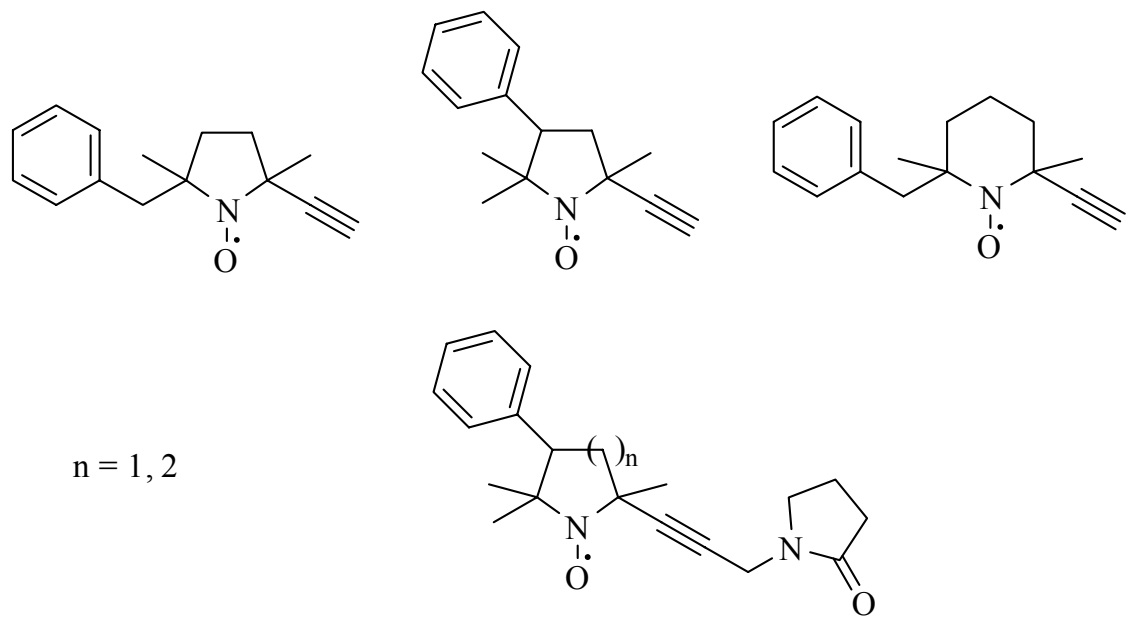

Figure 3 
Treatment of 2,5-dimethyl-3,4-dihydro-2H-pyrrole 1-oxide 1 or 2,6-dimethyl-2,3,4,5tetrahydro-pyridine 1-oxide 6 with benzylmagnesium chloride in dry $\mathrm{Et}_{2} \mathrm{O}$ followed by the oxidation of $\mathrm{N}$-hydroxy compound with activated $\mathrm{MnO}_{2}$ afforded the appropriate nitrones 2, 7 . The reaction of ethynylmagnesium bromide with 2-benzyl substituted nitrones 2 and 7 in THF yielded the stereoisomers of nitroxide $\mathbf{3}$ and $\mathbf{8}$, respectively. The ratio of trans/cis isomers was approximately 5:1. For further reactions the trans isomers were used. Complete assignments of compound 3 could be obtained with conventional 1D and 2D NMR spectroscopy methods after the treatment of the free radical compound with diphenylhydrazine in $\mathrm{CDCl}_{3}$.

The Grignard reaction of nitrone 11 with ethynylmagnesium bromide yielded 3-phenyl-5ethynyl substituted nitroxide 12. In the latter case the spacer between the nitrogen and aryl group is more rigid, being a part of a five-membered ring. To get more Deprenyl-like nitroxides $\mathbf{3}, \mathbf{8}$ and 12 were reduced to secondary amines by treating them with 5 equiv Fe powder in glacial acetic acid at $60{ }^{\circ} \mathrm{C}$ producing amines 4,9 and 13. These secondary amines were alkylated by refluxing them with methyl iodide excess in THF affording tertiary amines 5, 10 and 14 (Scheme 1). The advantages of synthesized secondary and tertiary amines beyond the structural fidelity are their better water solubility and oxygen scavenging ability such detoxyfying ROS during non-toxic, stable nitroxide free radical formation.

The oxidation of both 5- and 6-membered sterically hindered tertiary amines was investigated in $\mathrm{MeOH}$ with $\mathrm{H}_{2} \mathrm{O}_{2}$. However, upon oxidation in case of compound $\mathbf{1 4}$ or model compound $15^{26}$ upon oxidation nitroxides 12 and $16^{27}$ were formed and because of tetramethyl group no $\mathrm{MPP}^{+}$-like product is possible even at harsh oxidation conditions.

For Oxotremorine analogue synthesis we prepared compound 17 from nitrone 11 and a Grignard-reagent generated from propargylic alcohol with 2 equiv. EtMgBr in situ. After the oxidation of hydroxylamine with catalytic amount $\mathrm{MnO}_{2} 17$ propargylic alcohol was converted to 18 bromo compound by treating mesylate with $\mathrm{LiBr}$ in acetone. This bromo compound was used to alkylate pyrrolidine or 2-pyrrolidinone to yield spin labelled tremorine 19 or oxotremorine 20 derivatives. Nitroxides 19 and 20 were reduced to sterically hindered secondary amines 21, 22 with Fe powder in $\mathrm{AcOH}$ as mentioned above.

To avoid the quaternary salt formation of tertiary amine of compounds 21 and 22 the $N$ methylation of secondary amines by refluxing with formaldehyde in the presence of formic acid, (Eschweiler-Clark conditions) $^{21}$ furnished the tertiary pyrrolidine derivatives $\mathbf{2 3}$ and $\mathbf{2 4}$ (Scheme 2). 

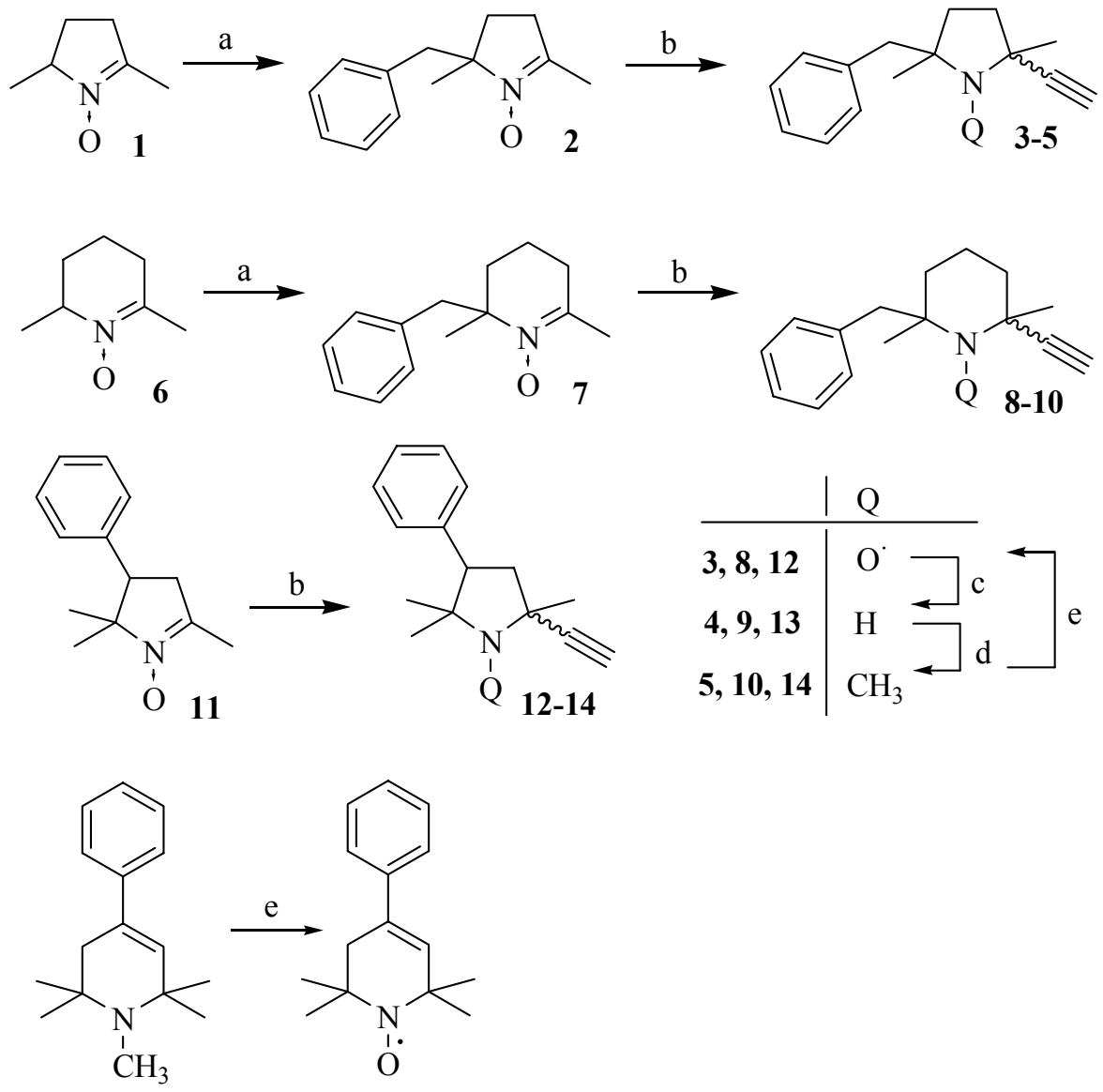

15

16

Scheme 1. Reagents and conditions: a: Benzylmagnesium chloride (1.2 equiv.), $\mathrm{Et}_{2} \mathrm{O}, 0{ }^{\circ} \mathrm{C} \rightarrow$ r.t., 3 h, aq. sat. $\mathrm{NH}_{4} \mathrm{Cl}$, then $\mathrm{CHCl}_{3}, \mathrm{MnO}_{2}$ (cat.), $\mathrm{O}_{2}$, r.t., 1 h (62-74 \%). B: $\mathrm{HC} \equiv \mathrm{CMgBr}, \mathrm{THF}, 0$ ${ }^{\circ} \mathrm{C} \rightarrow$ r.t., 2 h, aq. sat. $\mathrm{NH}_{4} \mathrm{Cl}$, then $\mathrm{CHCl}_{3}, \mathrm{MnO}_{2}$ (cat.), $\mathrm{O}_{2}$, r.t., 30 min (65-77 \%). C: $\mathrm{AcOH}, \mathrm{Fe}$ powder (5 equiv.), $60{ }^{\circ} \mathrm{C}, 1 \mathrm{~h}$, then $\mathrm{H}_{2} \mathrm{O}, \mathrm{K}_{2} \mathrm{CO}_{3}$ (57-68 \%). d: MeI, THF, reflux, $1 \mathrm{~h}$ (65-83 \%). (e) $\mathrm{H}_{2} \mathrm{O}_{2}$ (2 equiv), $\mathrm{Na}_{2} \mathrm{WO}_{4}$ (cat.), $\mathrm{MeOH}$, r.t., 24 h (35-42\%) 


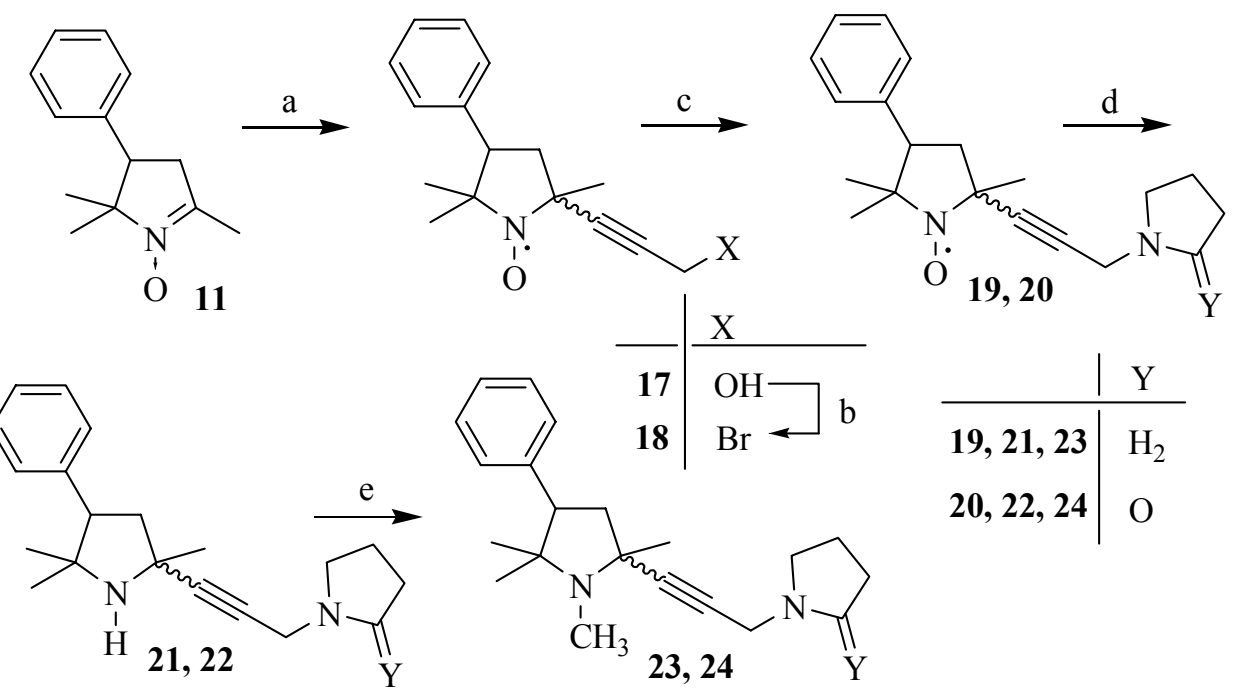

Scheme 2. Reagents and conditions: $\mathrm{a}: \mathrm{BrMgC} \equiv \mathrm{C}-\mathrm{CH}_{2}-\mathrm{OMgBr}$ (1.1 equiv.), $\mathrm{THF}, 0{ }^{\circ} \mathrm{C} \rightarrow$ r.t., 12 h, aq. sat. $\mathrm{NH}_{4} \mathrm{Cl}$, then $\mathrm{CHCl}_{3}, \mathrm{MnO}_{2}$ (cat.), $\mathrm{O}_{2}$, r.t., $1 \mathrm{~h}\left(58 \%\right.$ ). b: $\mathrm{Et}_{3} \mathrm{~N}, \mathrm{CH}_{2} \mathrm{Cl}_{2}, \mathrm{CH}_{3} \mathrm{SO}_{2} \mathrm{Cl}$, $0{ }^{\circ} \mathrm{C} \rightarrow$ r.t., $30 \mathrm{~min}$, then acetone, $\mathrm{LiBr}(2$ equiv.), reflux, $30 \mathrm{~min}(83 \%)$. c: pyrrolidine $(2$ equiv.), THF, reflux, $1 \mathrm{~h}$ (88 \% for 19); NaH, THF/DMF, 2-pyrrolidinone, r.t., $2.5 \mathrm{~h}$ (74\% for 20). d: $\mathrm{AcOH}, \mathrm{Fe}$ powder (5 equiv.), $60{ }^{\circ} \mathrm{C}, 1 \mathrm{~h}$ then $\mathrm{H}_{2} \mathrm{O}, \mathrm{K}_{2} \mathrm{CO}_{3}(53-71 \%)$. e: $\mathrm{HCHO}(37 \%$, 10 equiv.), $\mathrm{HCOOH}\left(88 \%\right.$, 10 equiv.), reflux, $6 \mathrm{~h}$, then $\mathrm{K}_{2} \mathrm{CO}_{3}(69-78 \%)$.

\section{Conclusions}

In conclusion, starting from disubstituted or trisubstituted five- or six-membered nitrones paramagnetic Deprenyl and Oxotremorine analogues were synthesized by means of Grignard reactions. Reduction of nitroxides and $\mathrm{N}$-methylation offered a closer analogue of Deprenyl and Oxotremorine neuroprotective drugs with ROS scavenging structural elements, such as sterically hindered amine (pre nitroxide) compounds. The biological study of these compounds is in progress and will be reported in due course.

\section{Experimental Section}

General. Melting points were determined with a Boetius micro melting point apparatus and are uncorrected. Elemental analyses $(\mathrm{C}, \mathrm{H}, \mathrm{N}, \mathrm{S})$ were performed on Fisons EA 1110 CHNS elemental analyzer. The IR (Specord 85) spectra were in each case consistent with the assigned structure. Mass spectra were recorded on a Thermoquest Automass Multi and VG TRIO-2 instruments in the EI mode. ${ }^{1} \mathrm{H}$ NMR spectra were recorded with Varian ${ }^{\text {UNITY }}$ INOVA $400 \mathrm{WB}$ spectrometer. Chemical shifts are referenced to $\mathrm{Me}_{4} \mathrm{Si}$. Measurements were run at $298 \mathrm{~K}$ probe temperature in $\mathrm{CDCl}_{3}$ solution. ESR spectra were taken on Miniscope MS 200 in $10^{-4} \mathrm{M} \mathrm{CHCl}_{3}$ 
solution and all monoradicals gave triplett spectrum $\mathrm{a}_{\mathrm{N}}=14.7-16.5$ G. Flash column chromatography was performed on Merck Kieselgel 60 (0.040-0.063 mm). Qualitative TLC was carried out on commercially available plates $(20 \times 20 \times 0.02 \mathrm{~cm})$ coated with Merck Kieselgel $\mathrm{GF}_{254}$.

\section{Synthesis of 2-benzyl-nitrones $(2,7)$; General procedure exemplified by 2-benzyl-2,5- dimethyl-3,4-dihydro-2H-pyrrole 1-oxide (2)}

To a solution of benzylmagnesium chloride (prepared from benzyl-chloride (7.62 g, $0.06 \mathrm{~mol}$ ) and $\mathrm{Mg}(1.50 \mathrm{~g}, 0.06 \mathrm{~mol}))$ in dry $\mathrm{Et}_{2} \mathrm{O}(50 \mathrm{~mL})$ nitrone $(\mathbf{1})(5.65 \mathrm{~g}, 0.05 \mathrm{~mol})$ in dry $\mathrm{Et}_{2} \mathrm{O}(50$ $\mathrm{mL}$ ) was added dropwise at $0{ }^{\circ} \mathrm{C}$. The reaction mixture was stirred at r.t. for $3 \mathrm{~h}$, then aq. sat. $\mathrm{NH}_{4} \mathrm{Cl}(80 \mathrm{~mL})$ was added. The organic layer was separated and the aqueous layer was extracted with $\mathrm{CHCl}_{3}(3 \times 30 \mathrm{~mL})$. The combined organic layer was dried $\left(\mathrm{MgSO}_{4}\right)$ and evaporated. The residue was dissolved in $\mathrm{CHCl}_{3}\left(50 \mathrm{~mL}\right.$ ), activated $\mathrm{MnO}_{2}$ (cat., $100 \mathrm{mg}$ ) was added and the mixture was bubbled with $\mathrm{O}_{2}$ for $1 \mathrm{~h}$ at r.t. The reaction mixture was then filtered, evaporated and purified by flash column chromatography with $\mathrm{CHCl}_{3} / \mathrm{MeOH}$ to give 2-benzyl-nitrone.

2-Benzyl-2,5-dimethyl-3,4-dihydro-2H-pyrrole 1-oxide (2). 7.51 g (74 \%); oil; $\mathrm{R}_{\mathrm{f}}=0.35$ $\left(\mathrm{CHCl}_{3}-\mathrm{Et}_{2} \mathrm{O}-\mathrm{MeOH}\right.$ 4:1.5:0.5); Anal. calcd. for $\mathrm{C}_{13} \mathrm{H}_{17} \mathrm{NO}$ (203.28): C 76.81\%, H 8.43\%, N $6.89 \%$; found: C $76.99 \%$, H $8.35 \%$, N $6.82 \%$. MS (EI) m/z (rel. int. \%): $203\left(\mathrm{M}^{+}, 63\right), 131$ (58), 112 (80), 91 (100).

2-Benzyl-2,6-dimethyl-2,3,4,5-tetrahydro-pyridine 1-oxide (7). $6.73 \mathrm{~g}$ (62\%); oil; $\mathrm{R}_{\mathrm{f}}=0.41$ $\left(\mathrm{CHCl}_{3}-\mathrm{Et}_{2} \mathrm{O}-\mathrm{MeOH}\right.$ 4:1.5:0.5); Anal. calcd. for $\mathrm{C}_{14} \mathrm{H}_{19} \mathrm{NO}$ (217.31): C 77.38\%, H 8.81\%, N $6.45 \%$; found: C $77.33 \%$, H $8.90 \%$, N $6.32 \%$. MS (EI) m/z (rel. int. \%): $217\left(\mathrm{M}^{+}, 10\right), 152$ (30), 129 (37), 91 (100), 73 (96), 41 (84).

Synthesis of 5- and 6-ethynyl-nitroxides $(3,8,12)$; General procedure exemplified by cisand trans-2-benzyl-5-ethynyl-2,5-dimethyl-pyrrolidine-1-yloxyl radical (3)

To ethynylmagnesium bromide $(0.5 \mathrm{M}$ sol. in THF, $50 \mathrm{~mL})$ a solution of nitrone (2) (4.06 g, 0.02 $\mathrm{mol})$ in dry THF $(30 \mathrm{~mL})$ was added dropwise at $0{ }^{\circ} \mathrm{C}$. The mixture was stirred at r.t. for $2 \mathrm{~h}$ then aq. sat. $\mathrm{NH}_{4} \mathrm{Cl}(40 \mathrm{~mL})$ was added. The organic layer was separated and the aqueous layer was extracted with $\mathrm{CHCl}_{3}(3 \times 20 \mathrm{~mL})$. The combined organic layer was dried $\left(\mathrm{MgSO}_{4}\right)$ and evaporated. The residue was dissolved in $\mathrm{CHCl}_{3}(50 \mathrm{~mL})$, activated $\mathrm{MnO}_{2}$ (cat., $100 \mathrm{mg}$ ) was added and the mixture was bubbled with $\mathrm{O}_{2}$ for $30 \mathrm{~min}$ at r.t. The reaction mixture was then filtered, evaporated and purified by flash column chromatography with hexane/Et ${ }_{2} \mathrm{O}$ to give the cis and trans stereoisomers of nitroxide.

Trans-2-benzyl-5-ethynyl-2,5-dimethyl-pyrrolidine-1-yloxyl Radical (trans-3). $2.78 \mathrm{~g}$ (61 $\%$ ); oil; $\mathrm{R}_{\mathrm{f}}=0.64$ (hexane-EtOAc 2:1); Anal. calcd. for $\mathrm{C}_{15} \mathrm{H}_{18} \mathrm{NO}$ (228.31): C 78.91\%, H 7.95 \%, N $6.13 \%$; found: C $78.89 \%$, H $7.82 \%$, N $6.31 \%$. MS (EI) m/z (rel. int. \%): 228 (M+22), 183 (5), 132 (17), 117 (95), 91 (100). 1H NMR ( $\mathrm{CDCl}_{3}+$ diphenyl-hydrazine) $\delta(\mathrm{ppm}): 2.78$ (d, $\left.J=12.5 \mathrm{~Hz}, 1 \mathrm{H}, \mathrm{Ph}-\mathrm{CH}_{2}\right), 2.70\left(\mathrm{~d}, J=12.5 \mathrm{~Hz}, 1 \mathrm{H}, \mathrm{Ph}-\mathrm{CH}_{2}\right), 2.47$ (s, 1H, $\left.-\mathrm{C} \equiv \mathrm{CH}\right), 1.97$ (m, 1H, $\left.3 \mathrm{~b}-\mathrm{CH}_{2}\right), 1.83\left(\mathrm{~m}, 2 \mathrm{H}, 4-\mathrm{CH}_{2}\right), 1.47\left(\mathrm{~s}, 3 \mathrm{H}, 2-\mathrm{CH}_{3}, 1,40\left(\mathrm{~m}, 1 \mathrm{H}, 3 \mathrm{a}-\mathrm{CH}_{2}\right), 1.33\left(\mathrm{~s}, 3 \mathrm{H}, 5-\mathrm{CH}_{3}\right)\right.$. 
Cis-2-benzyl-5-ethynyl-2,5-dimethyl-pyrrolidine-1-yloxyl Radical (cis-3). 730 mg (16 \%); oil; $\mathrm{R}_{\mathrm{f}}=0.57$ (hexane-EtOAc 2:1); Anal. calcd. for $\mathrm{C}_{15} \mathrm{H}_{18} \mathrm{NO}(228.31)$ : C $78.91 \%, \mathrm{H} 7.95 \%, \mathrm{~N}$ $6.13 \%$; found: C $78.72 \%$, H $7.89 \%$, N $6.24 \%$. MS (EI) m/z (rel. int. \%): $228\left(\mathrm{M}^{+}, 12\right), 183$ (5), 132 (18), 117 (59), 91 (100). 1H NMR $\left(\mathrm{CDCl}_{3}+\right.$ diphenyl-hydrazine) $\delta(\mathrm{ppm}): 3.24(\mathrm{~d}, J=12.3$ $\left.\mathrm{Hz}, 1 \mathrm{H}, \mathrm{Ph}-\mathrm{CH}_{2}\right), 3.02$ (d, J=12.3 Hz, 1H, $\left.\mathrm{Ph}-\mathrm{CH}_{2}\right), 2.53(\mathrm{~s}, 1 \mathrm{H},-\mathrm{C} \equiv \mathrm{CH}), 2.24\left(\mathrm{~m}, 1 \mathrm{H}, 3 \mathrm{~b}-\mathrm{CH}_{2}\right)$, $2.12\left(\mathrm{~m}, 1 \mathrm{H}, 4 \mathrm{~b}-\mathrm{CH}_{2}\right), 1.82\left(\mathrm{~m}, 1 \mathrm{H}, 3 \mathrm{a}-\mathrm{CH}_{2}\right), 1.56\left(\mathrm{~s}, 3 \mathrm{H}, 2-\mathrm{CH}_{3}\right), 1.36\left(\mathrm{~m}, 1 \mathrm{H}, 4 \mathrm{a}-\mathrm{CH}_{2}\right), 1.19$ $\left(\mathrm{s}, 3 \mathrm{H}, 5-\mathrm{CH}_{3}\right)$. To determine the orientation of the different groups in the molecule the NOESY experiment was used. The cross peaks within $\mathrm{H}(3 \mathrm{a}): 2-\mathrm{CH}_{3}$ and $2-\mathrm{CH}_{3}: 5-\mathrm{CH}_{3}$ suggest, that these groups are on the same side of the pyrrolidine ring.

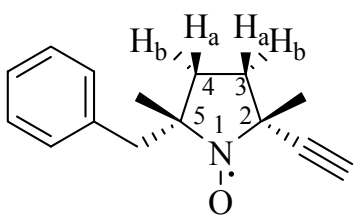

trans-2-Benzyl-6-ethynyl-2,6-dimethyl-piperidine-1-yloxyl radical (trans-8). $2.66 \mathrm{~g}$ (55 \%); thick oil; $\mathrm{R}_{\mathrm{f}}=0.66$ (hexane-EtOAc 2:1); Anal. calcd. for $\mathrm{C}_{16} \mathrm{H}_{20} \mathrm{NO}$ (242.34): C 79.30\%, H 8.32 \%, N $5.78 \%$; found: C $79.27 \%$, H $8.50 \%$, N 5.77 \%. MS (EI) m/z (rel. int. \%): $242\left(\mathrm{M}^{+}, 26\right)$, 152 (9), 117 (22), 91 (100).

cis-2-Benzyl-6-ethynyl-2,6-dimethyl-piperidine-1-yloxyl radical (cis-8). 485 mg (10\%); oil; $\mathrm{R}_{\mathrm{f}}=0.62$ (hexane-EtOAc 2:1); Anal. calcd. for $\mathrm{C}_{16} \mathrm{H}_{20} \mathrm{NO}$ (242.34): C 79.30\%, H 8.32\%, N $5.78 \%$; found: C $79.36 \%$, H $8.17 \%$, N $5.63 \%$. MS (EI) m/z (rel. int. \%): $242\left(\mathrm{M}^{+}, 35\right), 152$ (51), 117 (39), 91 (100).

trans-5-Ethynyl-2,2,5-trimethyl-3-phenyl-pyrrolidine-1-yloxyl radical (trans-12). $2.74 \mathrm{~g}$ (60 $\%$ ); mp 82-84 ${ }^{\circ} \mathrm{C} ; \mathrm{R}_{\mathrm{f}}=0.55$ (hexane-EtOAc 2:1); Anal. calcd. for $\mathrm{C}_{15} \mathrm{H}_{18} \mathrm{NO}$ (228.31): $\mathrm{C} 78.91$ \%, H $7.95 \%$, N $6.13 \%$; found: C $78.89 \%$, H 8.01 \%, N $6.10 \%$. MS (EI) m/z (rel. int. \%): 228 $\left(\mathrm{M}^{+}, 3\right), 183$ (6), 132 (61), 117 (100), 91 (72).

cis-5-Ethynyl-2,2,5-trimethyl-3-phenyl-pyrrolidine-1-yloxyl Radical (cis-12). 502 mg (11 \%); mp 140-141 ${ }^{\circ} \mathrm{C} ; \mathrm{R}_{\mathrm{f}}=0.51$ (hexane-EtOAc 2:1); Anal. calcd. for $\mathrm{C}_{15} \mathrm{H}_{18} \mathrm{NO}$ (228.31): $\mathrm{C} 78.91 \%$, H $7.95 \%$, N $6.13 \%$; found: C $78.77 \%$, H $7.81 \%$, N $5.96 \%$. MS (EI) m/z (rel. int. \%): 228 (M , 4), 183 (5), 132 (61), 117 (100), 91 (73).

\section{Synthesis of 5-(3-hydroxy-prop-1-ynyl)-2,2,5-trimethyl-3-phenyl-pyrrolidin-1-yloxyl radical} (17)

To a stirred solution of propargyl magnesium bromide $(0.022 \mathrm{~mol})$ (prepared from propargyl alcohol and 2 equiv. of ethyl magnesium bromide) in THF (50 mL) was added dropwise a solution of nitrone $11(4.06 \mathrm{~g}, 0.02 \mathrm{~mol})$ in THF $(20 \mathrm{~mL})$ at $0{ }^{\circ} \mathrm{C}$. After stirring the mixture at r.t. for $12 \mathrm{~h}$ sat. aq. $\mathrm{NH}_{4} \mathrm{Cl}(50 \mathrm{~mL})$ was added. The organic phase was separated, and the aqueous layer was extracted with $\mathrm{CHCl}_{3}$. The combined organic layer was dried $\left(\mathrm{MgSO}_{4}\right)$ and evaporated. The residue was dissolved in $\mathrm{CHCl}_{3}\left(20 \mathrm{~mL}\right.$ ), activated $\mathrm{MnO}_{2}$ (cat. $100 \mathrm{mg}$ ) was added and $\mathrm{O}_{2}$ 
was bubbled for $1 \mathrm{~h}$ at r.t. The mixture was filtered, evaporated and purified by flash column chromatography using hexane/EtOAc to give nitroxide 17, $2.99 \mathrm{~g}(58 \%)$; oil; $\mathrm{R}_{\mathrm{f}}=0.19\left(\mathrm{CHCl}_{3}\right.$ $\mathrm{Et}_{2} \mathrm{O}$ 2:1); Anal. calcd. for $\mathrm{C}_{16} \mathrm{H}_{20} \mathrm{NO}_{2}$ (258.34): C $74.39 \%$, H $7.80 \%$, N $5.42 \%$; found: C 74.28 \%, H $7.85 \%$, N 5.36 \%. MS (EI) m/z (rel. int. \%): $258\left(\mathrm{M}^{+}, 4\right), 243$ (15), 131 (17), 124 (57), 42 (100).

\section{Synthesis of 5-(3-bromo-prop-1-ynyl)-2,2,5-trimethyl-3-phenyl-pyrrolidin-1-yloxyl radical} (18)

To a stirred solution of nitroxide propargyl alcohol 17 (2.58 g, $0.01 \mathrm{~mol})$ and $\mathrm{Et}_{3} \mathrm{~N}(1.11 \mathrm{~g}, 1.10$ mol) in anhyd. $\mathrm{CH}_{2} \mathrm{Cl}_{2}(40 \mathrm{~mL}) \mathrm{CH}_{3} \mathrm{SO}_{2} \mathrm{Cl}(1.26 \mathrm{~g}, 1.10 \mathrm{~mol})$ was added dropwise at $0{ }^{\circ} \mathrm{C}$. The reaction mixture was stirred at r.t. for $30 \mathrm{~min}$ then the solvent was evaporated. The residue was dissolved in acetone $(20 \mathrm{~mL}), \operatorname{LiBr}(1.74 \mathrm{~g}, 0.02 \mathrm{~mol})$ was added and refluxed for $30 \mathrm{~min}$. Acetone was then evaporated, the residue was dissolved in $\mathrm{Et}_{2} \mathrm{O}$, washed with sat. aq. $\mathrm{NaCl}$, dried $\left(\mathrm{MgSO}_{4}\right)$ and evaporated. Propargyl bromide 18 was purified by flash column chromatography using hexane $/ \mathrm{Et}_{2} \mathrm{O}$ to yield: $2.66 \mathrm{~g}\left(83 \%\right.$ ); $\mathrm{mp} 61-63{ }^{\circ} \mathrm{C}$; $\mathrm{R}_{\mathrm{f}}=0.78$ (hexaneEtOAc 2:1); Anal. calcd. for $\mathrm{C}_{16} \mathrm{H}_{19} \mathrm{BrNO}$ (321.23): C 59.82 \%, H 5.96 \%, N $4.36 \%$, Br 24.87 \%; found: C 59.88 \%, H 5.89 \%, N 4.23 \%, Br $24.72 \%$. MS (EI) m/z (rel. int. \%): 322/320 (M , 16/16), 247/249 (15/15), 211 (32), 132 (100), 117 (95).

\section{Synthesis of 2,2,5-trimethyl-3-phenyl-5-(3-pyrrolidin-1-yl-prop-1-ynyl)-pyrrolidin-1-yloxyl radical (19)}

To a solution of propargyl bromide $18(2.57 \mathrm{~g}, 8.0 \mathrm{mmol})$ in dry THF $(30 \mathrm{~mL})$ pyrrolidine $(1.14$ $\mathrm{g}, 16 \mathrm{mmol}$ ) was added and the reaction mixture was boiled for $1 \mathrm{~h}$. The organic phase was washed with aq. sat. $\mathrm{NaCl}$, dried and purified by flash column chromatography with $\mathrm{CHCl}_{3} / \mathrm{Et}_{2} \mathrm{O}$ to yield pyrrolidine 19, $2.19 \mathrm{~g}(88 \%)$; oil; $\mathrm{R}_{\mathrm{f}}=0.19\left(\mathrm{CHCl}_{3}-\mathrm{Et}_{2} \mathrm{O} 2: 1\right)$; Anal. calcd. for $\mathrm{C}_{20} \mathrm{H}_{27} \mathrm{~N}_{2} \mathrm{O}$ (311.44): C 77.13\%, H 8.74 \%, N $8.99 \%$; found: C 77.28 \%, H $8.92 \%$, N $9.04 \%$. MS (EI) m/z (rel. int. \%): $311\left(\mathrm{M}^{+}, 33\right), 296$ (10), 108 (98), 84 (100).

\section{Synthesis of 1-[3-(1-oxyl-2,5,5-trimethyl-4-phenyl-pyrrolidin-2-yl)-prop-2-ynyl]-pyrrolidin-} 2-one radical (20)

To a suspension of $\mathrm{NaH}(72 \mathrm{mg}, 3 \mathrm{mmol})$ in dry THF (10 mL) DMF (3 mL) and 2-pyrrolidinone ( $255 \mathrm{mg}, 3 \mathrm{mmol}$ ) were added. The reaction mixture was stirred for $30 \mathrm{~min}$ then bromide 18 (963 $\mathrm{mg}, 3 \mathrm{mmol})$ was added dropwise in dry THF $(10 \mathrm{~mL})$. After stirring for $2 \mathrm{~h}$, sat. aq. $\mathrm{NaCl}$ was added $(10 \mathrm{~mL})$. The organic phase was separated, dried $\left(\mathrm{MgSO}_{4}\right)$ and evaporated. The resulting product was purified by flash chromatography to give $\mathbf{2 0}$ as yellow crystals: $722 \mathrm{mg}$ (74 \%); mp: 110-112 ${ }^{\circ} \mathrm{C} ; \mathrm{R}_{\mathrm{f}}=0.21\left(\mathrm{CHCl}_{3}-\mathrm{Et}_{2} \mathrm{O} 2: 1\right)$; Anal. calcd. for $\mathrm{C}_{20} \mathrm{H}_{25} \mathrm{~N}_{2} \mathrm{O}_{2}$ (325.43): $\mathrm{C} 73.82 \%, \mathrm{H}$ $7.74 \%$, N $8.61 \%$; found: C $73.84 \%$, H $7.67 \%$, N $8.52 \%$. MS (EI) m/z (rel. int. \%): $325\left(\mathrm{M}^{+}\right.$, 20), 311 (11), 280 (16), 210 (17), 195 (30), 164 (57), 132 (100), 98 (78), 91 (58). 
Reduction of nitroxides $(3,8,12,19,20)$ to sterically hindered secondary amines $(4,9,13$, 21, 22); General procedure exemplified by trans-2-benzyl-5-ethynyl-2,5-dimethylpyrrolidine (4)

To a solution of nitroxide $3(1.14 \mathrm{~g}, 5 \mathrm{mmol})$ in $\mathrm{AcOH}(20 \mathrm{~mL}) \mathrm{Fe}$ powder $(1.40 \mathrm{~g}, 25 \mathrm{mmol})$ was added, the mixture was warmed up to $60{ }^{\circ} \mathrm{C}$ and kept at this temperature for $1 \mathrm{~h}$. Then the reaction mixture was cooled to r.t., water $(30 \mathrm{~mL})$ was added and the solution was made alkaline with solid $\mathrm{K}_{2} \mathrm{CO}_{3}$. The aqueous phase was extracted with $\mathrm{CHCl}_{3}(3 \times 20 \mathrm{~mL}$ ). The organic layer was dried $\left(\mathrm{MgSO}_{4}\right)$ and evaporated. The residue was purified by flash column chromatography with $\mathrm{CHCl}_{3} / \mathrm{MeOH}$ to give amine.

trans-2-Benzyl-5-ethynyl-2,5-dimethyl-pyrrolidine (4). $725 \mathrm{mg}$ (68 \%); mp (HCl salt) 218-220 ${ }^{\circ} \mathrm{C} ; \mathrm{R}_{\mathrm{f}}=0.61\left(\mathrm{CHCl}_{3}-\mathrm{Et}_{2} \mathrm{O} 2: 1\right)$; Anal. calcd. for $\mathrm{HCl}$ salt $\mathrm{C}_{15} \mathrm{H}_{20} \mathrm{NCl}$ (249.78): $\mathrm{C} 72.13 \%, \mathrm{H}$ $8.07 \%$, N $5.61 \%, \mathrm{Cl} 14.19 \%$; found: C $72.30 \%, \mathrm{H} 8.21 \%, \mathrm{~N} 5.73 \%$, Cl 14.25\%. MS (EI) m/z (rel. int. \%): $213\left(\mathrm{M}^{+}, 28\right), 198$ (1), 122 (100), 91 (93).

trans-2-Benzyl-6-ethynyl-2,6-dimethyl-piperidine (9). $648 \mathrm{mg}$ (57\%); mp (HCl salt) 225-227 ${ }^{\circ} \mathrm{C} ; \mathrm{R}_{\mathrm{f}}=0.52\left(\mathrm{CHCl}_{3}-\mathrm{Et}_{2} \mathrm{O} 2: 1\right)$; Anal. calcd. for $\mathrm{HCl}$ salt $\mathrm{C}_{16} \mathrm{H}_{22} \mathrm{NCl}$ (263.81): $\mathrm{C} 72.85 \%, \mathrm{H}$ $8.41 \%$, N $5.31 \%$, Cl 13.44 \%; found: C $72.76 \%, \mathrm{H} 8.53 \%$, N $5.39 \%$, Cl 13.60\%. MS (EI) m/z (rel. int. \%): $227\left(\mathrm{M}^{+}, 1\right), 212$ (21), 136 (100), 91 (98), 77 (77), 42 (98).

trans-5-ethynyl-2,2,5-trimethyl-3-phenyl-pirrolidine (13). $661 \mathrm{mg}$ (62\%); mp ( $\mathrm{HCl}$ salt) sublimates over $220{ }^{\circ} \mathrm{C} ; \mathrm{R}_{\mathrm{f}}=0.55\left(\mathrm{CHCl}_{3}-\mathrm{Et}_{2} \mathrm{O}-\mathrm{MeOH} 4: 1.5: 0.5\right)$; Anal. calcd. for $\mathrm{HCl}$ salt $\mathrm{C}_{15} \mathrm{H}_{20} \mathrm{NCl}$ (249.78): C 72.13\%, H $8.07 \%$, N 5.61 \%, Cl 14.19\%; found: $\mathrm{C} 72.15 \%$, H $7.96 \%$, N $5.65 \%, \mathrm{Cl} 14.22 \%$. MS (EI) m/z (rel. int. \%): $213\left(\mathrm{M}^{+}, 15\right), 198$ (20), 141 (31), 109 (100), 94 (65), 42 (28).

2,2,5-Trimethyl-3-phenyl-5-(3-pyrrolidin-1-yl-prop-1-ynyl)-pyrrolidine (21). $786 \mathrm{mg}$ (53 \%); mp 140-142 ${ }^{\circ} \mathrm{C} ; \mathrm{R}_{\mathrm{f}}=0.11\left(\mathrm{CHCl}_{3}-\mathrm{Et}_{2} \mathrm{O}-\mathrm{MeOH} 4: 1.5: 0.5\right)$; Anal. calcd. for $\mathrm{C}_{20} \mathrm{H}_{28} \mathrm{~N}_{2}$ (296.45): C $81.03 \%$, H $9.52 \%$, N $9.45 \%$; found: C $81.02 \%$, H $9.55 \%$, N $9.58 \%$. MS (EI) m/z (rel. int. \%): $296\left(\mathrm{M}^{+}, 4\right), 281$ (3), 227 (19), 121 (68), 91 (22), 81 (36), 69 (100).

1-[3-(2,5,5-Trimethyl-4-phenyl-pyrrolidin-2-yl)-prop-2-ynyl]-pyrrolidin-2-one (22). $1.102 \mathrm{~g}$ (71 \%); oil; $\mathrm{R}_{\mathrm{f}}=0.21\left(\mathrm{CHCl}_{3}-\mathrm{Et}_{2} \mathrm{O}-\mathrm{MeOH} 4: 1.5: 0.5\right)$; Anal. calcd. for $\mathrm{C}_{20} \mathrm{H}_{26} \mathrm{~N}_{2} \mathrm{O}$ (310.44): C $77.38 \%$, H $8.44 \%$, N $9.02 \%$; found: C $77.49 \%$, H $8.41 \%$, N $9.08 \%$. MS (EI) m/z (rel. int. \%): $310\left(\mathrm{M}^{+}, 1\right), 295$ (2), $206(13), 98$ (58), 42 (100).

Alkylation of secondary amines $(4,9,13)$ to $N$-methyl derivatives $(5,10,14)$. General procedure exemplified by trans-2-benzyl-5-ethynyl-1,2,5-trimethyl-pyrrolidine (5)

To a solution of amine 4 (213 mg, $1 \mathrm{mmol})$ in THF (15 mL) MeI (142 mg, $1 \mathrm{mmol})$ was added and the reaction mixture was refluxed for $1 \mathrm{~h}$. The organic phase was extracted with $10 \% \mathrm{~K}_{2} \mathrm{CO}_{3}$ solution, dried $\left(\mathrm{MgSO}_{4}\right)$ and evaporated. The residue was purified by flash chromatography with $\mathrm{CHCl}_{3} / \mathrm{Et}_{2} \mathrm{O}$ as eluent to give $N$-methyl derivative.

trans-2-Benzyl-5-ethynyl-1,2,5-trimethyl-pyrrolidine (5). $188 \mathrm{mg}$ (83 \%); mp (HCl salt) sublimates over $185{ }^{\circ} \mathrm{C} ; \mathrm{R}_{\mathrm{f}}=0.85\left(\mathrm{CHCl}_{3}-\mathrm{Et}_{2} \mathrm{O} 2: 1\right)$; Anal. calcd. for $\mathrm{HCl}$ salt $\mathrm{C}_{16} \mathrm{H}_{22} \mathrm{NCl}$ 
(263.81): C $72.85 \%$, H $8.41 \%$, N $5.31 \%, \mathrm{Cl} 13.44 \%$; found: C $72.77 \%$, H $8.47 \%$, N $5.20 \%$, $\mathrm{Cl} 13.49 \%$. MS (EI) m/z (rel. int. \%): $227\left(\mathrm{M}^{+}, 28\right), 212$ (2), 202 (2), 136 (100), 91 (93).

trans-2-Benzyl-6-ethynyl-1,2,6-trimethyl-piperidine (10). $157 \mathrm{mg}$ (65\%); mp (HCl salt) sublimates over $195{ }^{\circ} \mathrm{C} ; \mathrm{R}_{\mathrm{f}}=0.46$ (hexane-EtOAc 2:1); Anal. calcd. for $\mathrm{HCl}$ salt $\mathrm{C}_{17} \mathrm{H}_{24} \mathrm{NCl}$ (277.84): C $73.49 \%$, H $8.71 \%$, N $5.04 \%$, Cl $12.76 \%$; found: C $73.55 \%$, H 8.62\%, N $4.97 \%$, $\mathrm{Cl} 12.61 \%$. MS (EI) m/z (rel. int. \%): MS (EI) m/z (rel. int. \%): $241\left(\mathrm{M}^{+}, 13\right), 226(8), 148$ (100), 91 (82).

trans-2-Ethynyl-1,2,5,5-tetramethyl-4-phenyl-pyrrolidine (14). $163 \mathrm{mg}$ (72\%); mp (HCl salt) 202-204 ${ }^{\circ} \mathrm{C} ; \mathrm{R}_{\mathrm{f}}=0.71\left(\mathrm{CHCl}_{3}-\mathrm{Et}_{2} \mathrm{O} 2: 1\right)$; Anal. calcd. for $\mathrm{HCl}$ salt $\mathrm{C}_{16} \mathrm{H}_{22} \mathrm{NCl}$ (263.81): $\mathrm{C} 72.85$ \%, H $8.41 \%$, N $5.31 \%$, Cl 13.44 \%; found: C $72.85 \%$, H $8.31 \%$, N $5.27 \%$, Cl 13.28\%. MS (EI) m/z (rel. int. \%): $227\left(\mathrm{M}^{+}, 7\right), 212$ (100), 186 (17), 108 (11), 91 (7), 56 (19).

\section{Oxidation of tertiary amines $(14,15)$ to nitroxides $(12,16)$}

To a solution of tertiary amine $14(227 \mathrm{mg}, 1 \mathrm{mmol})$ or $\mathbf{1 5}(229 \mathrm{mg}, 1 \mathrm{mmol})$ in $\mathrm{MeOH}(5 \mathrm{~mL})$ $\mathrm{H}_{2} \mathrm{O}_{2}(30 \%$ sol. in water, $0.5 \mathrm{~mL}, 5 \mathrm{mmol})$ and $\mathrm{Na}_{2} \mathrm{WO}_{4}$ (cat, $10 \mathrm{mg}$ ) was added at r.t. The reaction mixture was allowed to stand for $24 \mathrm{~h}$ at this temperature. The solvent was evaporated, brine $(5 \mathrm{~mL})$ was added and the aqueous phase was extracted with $\mathrm{CHCl}_{3}(3 \mathrm{x} 5 \mathrm{~mL})$. The organic phase was dried $\left(\mathrm{MgSO}_{4}\right)$, evaporated and purified with flash column chromatography to yield nitroxides 12 (96 mg, $42 \%$ ) and 16 (80 mg, $35 \%)$. The physical and spectroscopic data of these compounds are identical described above.

Synthesis of 1,2,2,5-tetramethyl-3-phenyl-5-(3-pyrrolidin-1-yl-prop-1-ynyl)-pyrrolidine (23) and 1,2,2,5-tetramethyl-3-phenyl-5-(3-pyrrolidin-1-yl-prop-1-ynyl)-pyrrolidin-2-one (24)

A mixture of the free base of 21 (296 mg, $1 \mathrm{mmol})$ or $22(310 \mathrm{mg}, 1 \mathrm{mmol})$ and 10-fold excess each of $37 \%$ formalin and $88 \%$ formic acid was heated at reflux for $6 \mathrm{~h}$. After cooling to room temperature, water $(5 \mathrm{~mL})$ was added and the aqueous phase was basified with solid $\mathrm{K}_{2} \mathrm{CO}_{3}$ and extracted with $\mathrm{CHCl}_{3}(3 \times 10 \mathrm{~mL})$. The organic phase was dried $\left(\mathrm{MgSO}_{4}\right)$ and concentrated under vacuum to give $N$-methyl derivatives.

1-[3-(1,2,5,5-Tetramethyl-4-phenyl-pyrrolidin-2-yl)-prop-2-ynyl]-pyrrolidine (23). $242 \mathrm{mg}$ (78 \%); oil; $\mathrm{R}_{\mathrm{f}}=0.28\left(\mathrm{CHCl}_{3}-\mathrm{Et}_{2} \mathrm{O}-\mathrm{MeOH}\right.$ 4:1.5:0.5); Anal. calcd. for $\mathrm{C}_{21} \mathrm{H}_{30} \mathrm{~N}_{2}$ (310.48): C $81.24 \%$, H 9.74 \%, N $9.02 \%$; found: C $81.17 \%$, H $9.82 \%$, N $8.96 \%$. MS (EI) m/z (rel. int. \%): $310\left(\mathrm{M}^{+}, 5\right), 295$ (92), $226(50), 91$ (69), 56 (100).

1-[3-(1,2,5,5-Tetramethyl-4-phenyl-pyrrolidin-2-yl)-prop-2-ynyl]-pyrrolidin-2-one (24). 223 mg (69 \%); oil; $\mathrm{R}_{\mathrm{f}}=0.23\left(\mathrm{CHCl}_{3}-\mathrm{Et}_{2} \mathrm{O} 2: 1\right)$; Anal. calcd. for $\mathrm{C}_{21} \mathrm{H}_{28} \mathrm{~N}_{2} \mathrm{O}$ (324.47): $\mathrm{C} 77.74 \%, \mathrm{H}$ $8.70 \%$, N $8.63 \%$; found: C $77.63 \%$, H $8.85 \%$, N $8.53 \%$. MS (EI) m/z (rel. int. \%): $324\left(\mathrm{M}^{+}\right.$, 2), 309 (81), 224 (34), 83 (100). 


\section{Acknowledgements}

This work was supported by a grant from the Hungarian National Research Fund (OTKA K81123 and M045190). The authors thank Krisztina Kish for the elemental analysis, to Viola H. Csokona and Noémi Lazsányi for technical assistance.

\section{References}

1. Jenner, P.; Orlanow, C.W., Pathological Evidence for Oxidative Stress in Parkinson's Disease and Related Degenerative Disorders, In Neurodegeneration and Neuroprotection in Parkinson's Disease, C. W. Orlanow, P. Jenner and M. Youdim, Eds., Academic Press, New York, 1996, pp 23-45.

2. Ehringer, H.; Hornykiewicz, O., Klin. Wochenschr. 1960, 38, 1236.

3. Kish, S. J.; Shannak, K.; Hornykiewicz, O., N. Engl. J. Med. 1988, 318, 876.

4. Vernier, V. G. Antiparkinsonism Drugs In Burger's Medicinal Chemistry and Drug Discovery, Wolff, M. E. Ed. Wiley: New York, 1996, Vol. 3, Ch. 37, pp. 43-93.

5. Ebadi, M.; Sharma, S.; Shavali, S., El Refaey, H. J. Neuoscience Res. 2002, 67, 285.

6. Mandel, S.; Grunblatt, E.; Riederer, P.; Gerlach, M.; Levites, Y.; Youdim, M. B. H., CNS Drugs 2003, 17, 729.

7. Gulyás, B.; Pavlova, E.; Kása, P.; Gulya, K.; Bakota, L.; Várszegi, Sz.; Keller, É.; Horváth, M. Cs.; Nag, S.; Hermecz, I.; Magyar, K.; Halldin, C. Neurochem. Int. 2011, 58, 60.

8. Magyar, K.; Pálfi, M.; Tábi, T.; Kalász, H.; Szende, B.; Szökö, E. Curr. Med. Chem. 2004, 11, 2017.

9. Carrillo, M. C.; Minami, C.; Kitani, K.; Maruyama, W.; Ohashi, K.; Yamamoto, T.; Naoi, M.; Kanai, S.; Youdim, M. B. H. Life Sci. 2000, 67, 577.

10. Gerlach, M.; Riederer, P. Youdim, M. B. H., Eur. J. Pharm. Mol. Pharm. 1992, $226,97$.

11. De Marchi, U.; Pietrangeli, P.; Marcocci, L.; Mondovi, B.; Toninello, A. Biochem. Pharmacol. 2003, 66, 1749.

12. Binda, C.; Hubálek, F.; Li, M.; Herzig, Y.; Sterling, J.; Edmondson, D. E.; Mattevi, A. J. Med. Chem. 2004, 47, 1767.

13. Namiecinski, M.; Pulaski, L.; Kochman, A.; Skolimowski, J.; Bartosz, G.; Metodiewa, D. in vivo, 2004, 18, 171.

14. Kochman, A.; Skolimowski, J.; Gebicka, L.; Metodiewa, D. Pol. J. Pharmacol. 2003, 55, 389.

15. Halmosi, R.; Deres, P.; Berente, Z.; Kálai, T.; Sümegi, B.; Hideg, K.; Tóth, K. J. Cardiovasc. Pharm. 2002, 40, 854.

16. Deres, P.; Halmosi, R.; Tóth, A.; Kovács, K.; Pálfi, A.; Habon, T.; Czopf, L.; Kálai, T.; Hideg, K.; Sümegi, B.; Tóth, K. J. Cardiovasc. Pharmacol. 2005, 45(1), 36. 
17. Kálai, T.; Várbíró, G.; Bognár, Z.; Pálfi, A.; Hantó, K.; Bognár, B.; Ösz, E.; Sümegi, B.; Hideg, K. Bioorg. Med. Chem. 2005, 13, 2629.

18. Kutala, V. K.; Khan, M.; Mandal, R.; Ganesan, L. P.; Tridandapani, S.; Kálai, T.; Hideg, K.; Kuppusamy, P. J. Pharm. Exp. Ther. 2006, 317, 921.

19. Kálai, T.; Khan, M.; Balog, M.; Kutala, K. V.; Kuppusamy, P.; Hideg, K. Bioorg. Med. Chem. 2006, 14, 5510.

20. Cannon, J. G. Cholinergics In Burger's Medicinal Chemistry and Drug Discovery, Wolff, M.E. Ed. Wiley: New York, 1996, Vol. 3, Ch. 45, pp. 28-36.

21. Garvey, D. S.; Wasicak, J. T.; Chung, J. Y.-L.; Shue, Y.-K.; Carrera, G. M.; May, P. D.; McKinney, M. M.; Anderson, D.; Cadman, E.; Vella-Rountree, L.; Nadzan, A.M.; Williams, M. J. Med. Chem. 1992, 35, 1550.

22. Chung, J. Y. L.; Wasicak, J. T. Tetrahedron Lett. 1990, 31, 3957.

23. Pretorius, A.; Ogunrombi, M. O.; Terre'Blanche, G..; Castagnoli Jr., N.; Bergh, J. J.; Petzer, J. P. Bioorg. Med. Chem. 2008, 16, 8813.

24. Grimm, M. L.; Allen, W. J.; Finn, M.; Castagnoli Jr., N.; Tanko, J. M. Bioorg. Med. Chem. 2011, 19, 1458.

25. Adams Jr., J. D. Agents Used in Neurodegenerative Disorders In Burger's Medicinal Chemistry and Drug Discovery, Wolff, M. E. Ed. Wiley, New York, 1996, Vol. 3, Ch. 40, pp. 261-319.

26. Beckett, A. H.; Casy, A. F.; Lingard, R. G.; Iorio, M. A. Hewitson, K. Tetrahedron 1966, 22, 2735.

27. Pavlikov, V. V.; Shapiro, A. B.; Rozantsev, E. G. Doklady Akedemii Nauk SSSR 1978, 242(2), 369; Chem. Abstr. 1979, 90, 72541. 